\title{
ALGEBRAS OF FUNCTIONS ON SEMITOPOLOGICAL LEFT-GROUPS
}

\author{
BY
}

JOHN F. BERGLUND AND PAUL MILNES $\left({ }^{\mathbf{1}}\right)$

\begin{abstract}
We consider various algebras of functions on a semitopological left-group $S=X \times G$, the direct product of a left-zero semigroup $X$ and a group $G$. In $\S 1$ we examine various analogues to the theorem of Eberlein that a weakly almost periodic function on a locally compact abelian group is uniformly continuous. Several appealing conjectures are shown by example to be false. In the second section we look at compactifications of products $S \times T$ of semitopological semigroups with right identity and left identity, respectively. We show that the almost periodic compactification of the product is the product of the almost periodic compactifications, thus generalizing a result of deLeeuw and Glicksberg. The weakly almost periodic compactification of the product is not the product of the weakly almost periodic compactifications except in restrictive circumstances; for instance, when $T$ is a compact group. Finally, as an application, we define and study analytic weakly almost periodic functions and derive the theorem, analogous to a classical theorem about almost periodic functions, that an analytic function which is weakly almost periodic on a single line is analytic weakly almost periodic on a whole strip.
\end{abstract}

Introduction. Semigroups of various types are the natural objects on which to study spaces of functions which are "almost periodic" in some sense or "uniformly continuous" in some sense. Much of the study of such function spaces has either concentrated on general theory with no algebraic restrictions being placed on the semigroups, or it has dealt with the special case in which the semigroup is, in fact, a group. We propose to look at a special type of semigroup, a left-group, which, although it is not a group, is yet rich enough in algebraic structure to allow a detailed analysis. A semitopological semigroup is a set $S$ together with an associative binary operation ("multiplication")

$$
(s, t) \rightarrow s t: S \times S \rightarrow S
$$

and a Hausdorff topology with respect to which the binary operation is separately continuous. If the multiplication is jointly continuous, then $S$ is called a topological semigroup. A semigroup is called a left-group if it is left simple

Received by the editors July 2, 1974.

AMS (MOS) subject classifications (1970). Primary 43A15, 22A20; Secondary 43A60, $46 \mathrm{~J} 15$.

Key words and phrases. Semitopological semigroup, left-group, weakly almost periodic function, compactification, analytic function.

( $\left.{ }^{1}\right)$ Supported in part by NRC Grant A7857. 
and right cancellative. A left-group always decomposes algebraically into a direct product $X \times G$ of a left-zero $\left(x_{1} x_{2}=x_{1}\right)$ semigroup $X$ and a group $G$. For a left-group which is also a locally compact semitopological semigroup, the direct product decomposition $X \times G$ is also topological, with $X$ a locally compact leftzero semigroup and $G$ a locally compact topological group [3, II.2.3]. However, the decomposition need not be topological. If the left-group $S=[0,1] \times R$, for example, is given the Knight-Moran-Pym topology of separate continuity [11], which makes $S$ a semitopological semigroup, then the direct product decomposition is not topological. To avoid this pathology here, we define a semitopological left-group to be a direct product $S=X \times G$ of a left-zero topological semigroup $X$ and a semitopological semigroup $G$ which is algebraically a group. [Note that, if $G$ is locally compact, then it must be a topological group and $S$ must be a topological semigroup.]

Let $S$ be a semitopological semigroup. Denote the $C^{*}$-algebra of all bounded, continuous complex-valued functions on $S$ by $C(S)$. Given $s \in S$, define the left translation operator $L_{s}$ [respectively, the right translation operator $R_{s}$ ] on $C(S)$ by

$$
L_{s} f(t)=f(s t), \quad t \in S, \quad\left[R_{s} f(t)=f(t s), t \in S\right]
$$

for each $f \in C(S)$. Let

$$
O_{L}(f)=\left\{L_{s} f \mid s \in S\right\}, \quad\left[O_{R}(f)=\left\{R_{s} f \mid s \in S\right\}\right] .
$$

A function $f \in C(S)$ is called almost periodic if $O_{R}(f)$ is relatively compact in the norm topology of $C(S)$; equivalently, $f$ is almost periodic if $O_{L}(f)$ is relatively norm compact in $C(S)$. Call $f \in C(S)$ weakly almost periodic if $O_{R}(f)$ is relatively compact in the weak topology of $C(S)$; equivalently, $f$ is weakly almost periodic if $O_{L}(f)$ is relatively weakly compact in $C(S)$. Denote the set of almost periodic functions on $S$ by $A P(S)$ and the set of weakly almost periodic functions by $W A P(S)$. Both $A P(S)$ and $W A P(S)$ are $C^{*}$-subalgebras of $C(S)$. Other $C^{*}$. subalgebras of $C(S)$ are defined as follows:

(1) A function $f \in C(S)$ is in $L U C(S)$ [respectively, $R U C(S)$ ] if the function

$$
s \rightarrow L_{s} f: S \rightarrow C(S), \quad\left[s \rightarrow R_{s} f: S \rightarrow C(S)\right]
$$

is continuous when $C(S)$ has its norm topology.

(2) A function $f \in C(S)$ is in $L M C(S)$ [respectively, $R M C(S)$ ] if $O_{R}(f)$ [respectively, $O_{L}(f)$ ] is relatively compact in the pointwise topology of $C(S)$. (The authors are preparing a comprehensive study of these last subspaces and related topics.)

The relationships among the various $C^{*}$-algebras defined above are given by the following diagram (all arrows are inclusions): 
(A)

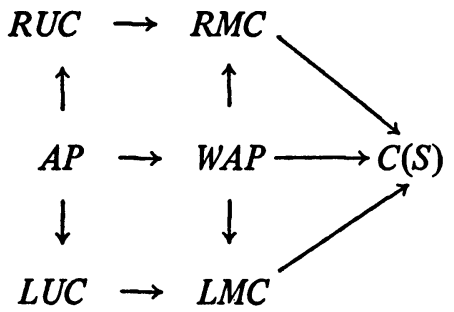

Comments about the notation $L M C$ and the inclusion $L U C \subset L M C$ are in order. (Analogous comments can be made about $R M C$ and $R U C$.) Let $\beta S$ be the spectrum of $C(S)$, which is just the Stone-Čech compactification of $S$ if $S$ is completely regular. Then $L M C$ turns out [12, Theorem 3.1$]$ to be equal to the left multiplicatively continuous subspace of Mitchell [13],

$\left\{f \in C(S) \mid\right.$ the function $s \rightarrow \mu\left(L_{s} f\right)$ is continuous for each $\left.\mu \in \beta S\right\}$, which obviously contains $L U C$.

In [12] an example is given of a locally compact semitopological semigroup $S$ for which all the inclusions of (A) are proper. In addition, neither $W A P(S) \subseteq$ $L U C(S)$ nor $L U C(S) \subseteq W A P(S)$. On the other hand, if $G$ is a locally compact group, then

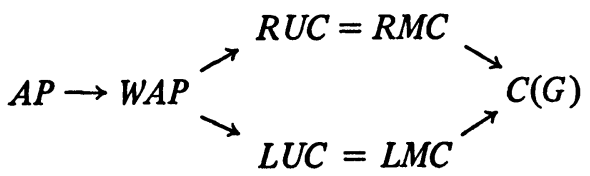

Moreover, $L U C(G)$ [respectively, $R U C(G)$ ] is the space of all bounded complexvalued functions on $G$ which are uniformly continuous with respect to the right [respectively, left] uniform structure on $G$ [4, Chapter III, §3].

A left-group is, in many ways, the simplest of nontrivial semigroups. (From a semigroup viewpoint, a group is "trivial".) One is naturally led, therefore, to considerations of left-groups when moving from statements about groups to statements about semigroups. Our interest in left-groups, however, does not stem solely from the desire to look at objects which are almost groups. Rather, the initial motive for studying these functions came from the observation that the bounded almost periodic functions depending uniformly on parameters of Corduneanu [5, II.1] are precisely almost periodic functions on topological left-groups $S=X \times G$, where $X$ is a subset of complex $n$-space $C^{n}$, and $G$ is the additive group of real numbers, provided that the parameter space $X$ is compact [5, Theorem 2.6, p. 54].

Several of the theorems of Corduneanu reduce to the statement that the set of almost periodic functions on $S=X \times G$ is a $C^{*}$-subalgebra of $C(S)$. Corduneanu also discussed the uniform continuity of almost periodic functions 
depending uniformly on parameters. In $\S 1$, we consider various analogues to Corduneanu's theorem. Besides $L U C(S)$ and $R U C(S)$, we also deal with actual uniform continuity: If $S=X \times G$ is a semitopological left-group with $X$ a uniform space and $G$ a topological group, let $U_{L}=U_{L}(S)$ be the space of all functions $f \in C(S)$ which are uniformly continuous when $G$ is given the uniformity with basis

$$
\left\{(s, t) \in G \times G \mid s t^{-1} \in V\right\}
$$

as $V$ runs through the neighbourhoods of the identity in $G$, and $S$ is given the product uniformity. Define $U_{R}=U_{R}(S)$ similarly using the left uniform structure on $G$. The spaces $U_{L}$ and $U_{R}$ are particularly natural to study when $X$ is compact and hence has a unique uniformity.

Corduneanu observed that Bohr's definition of analytic almost periodic functions may be phrased in terms of almost periodic functions depending uniformly on parameters. Consequently, the definition may be phrased in terms of almost periodic functions on left-groups; and we are led naturally to various spaces of analytic functions. The only one of interest, it turns out, besides the analytic almost periodic functions, is the space of analytic weakly almost periodic functions, which we study in $\$ 3$.

Each section contains a summary of the results of that section.

1. Inclusion relationships: General theorems and counterexamples. In [12 p. 502, Example] it was shown that the orderly sequence of inclusions and equal. ities that holds among the subspaces under consideration for locally compact groups [see (B) in the Introduction] breaks down to a large extent for semitopological semigroups; the semigroup of the example in [12] is explicitly assumed not to be topological. We show in this section that much of this breakdown can occur for topological semigroups, even for ones of very simple type, the locally compact topological left-groups. Some positive results are proved first. We remark that, in the following theorem, the hypothesis that $X$ is a uniform space is required only for (i), (ii) and one assertion in (iv).

THEOREM 1.1. Let $S=X \times G$ be a topological left-group such that $X$ is a uniform space with uniformity $U$.

(i) $L U C \supset U_{L}$ and $L U C=U_{L}$ if $X$ is compact.

(ii) RUC $\supset U_{R}$ and the containment can be proper even if $X$ is compact.

(iii) $f \in R U C$ if and only if $f \in C(S)$ and the set of functions

$$
A_{f}=\left\{F_{x} \mid x \in X\right\} \subset C(G)
$$

where 


$$
F_{x}(s)=f(x, s) \text { for all } s \in G,
$$

is left uniformly equicontinuous.

(iv) If $\psi: S \rightarrow S$ is defined by $\psi(x, s)=\left(x, s^{-1}\right)$, then the adjoint $\psi^{*}$ effects an isometric isomorphism of $U_{L}$ onto $U_{R}$, of WAP onto itself, and of $A P$

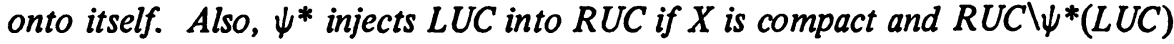
can be nonvoid even if $X$ is compact.

Proof. (i) It follows immediately from definitions that $L U C \supset U_{L}$. Sup. pose $X$ is compact and $f \in L U C$. We must show $f \in U_{L}$, i.e., given $\epsilon>0$, we must find $U \in U$ and a neighbourhood $V$ of the identity $e$ of $G$ such that

$$
|f(x, u)-f(y, t)|<\epsilon
$$

whenever $(x, y) \in U, u t^{-1} \in V$. We do this as follows. Given any $(x, s) \in S$, we can find a neighbourhood $U_{x} \times V_{s}$ of $(x, s)$, where $U_{x}$ and $V_{s}$ are neighbourhoods of $x$ and $s$, respectively, such that

$$
|f(x, s r)-f(y, t r)|<\epsilon / 2
$$

for all $r \in G$ whenever $(y, t) \in U_{x} \times V_{s}$. Thus

$$
|f(x, u)-f(y, t)|<\epsilon / 2
$$

whenever $y \in U_{x}$ and $u t^{-1} \in s V_{s}^{-1}$. Since $X$ is compact,

$$
X=\bigcup_{1}^{n} U_{x_{i}}
$$

for a suitably chosen $x_{i}, i=1,2, \ldots, n$. Also, there is a $U \in U$ such that, for each $y \in X$,

$$
U(y)=\left\{y^{\prime} \mid\left(y, y^{\prime}\right) \in U\right\} \subset U_{x_{i}}
$$

for some $i$. Then, if $(x, y) \in U$ and $u t^{-1} \in V=\bigcap_{1}^{n} s_{i} V_{s_{i}}^{-1}$,

$$
|f(x, u)-f(y, t)| \leqslant\left|f(x, u)-f\left(x_{i}, t\right)\right|+\left|f\left(x_{i}, t\right)-f(y, t)\right|<\epsilon
$$

for at least one $i$ as required. [See the remark following the proof of this theorem for a left-group $S=X \times G$ with $X$ not compact and $L U C \backslash U_{L} \neq \varnothing$.]

(ii) The fact that $R U C \supset U_{R}$ also follows immediately from definitions. A function in $R U C \backslash U_{R}$ is constructed on the left-group $[0,1] \times R$ following Theorem 1.4 ahead.

(iii) $f \in R U C$ if and only if $f$ is continuous and

$$
\lim \sup \left\{\left|f\left(x, \operatorname{tr}_{\alpha}\right)-f(x, \operatorname{tr})\right| \mid(x, t) \in S\right\}=0
$$


whenever $\left\{r_{\alpha}\right\}$ converges to $r \in G$. The desired conclusion follows directly from this assertion and the fact that, for a topological group $G, R U C(G)$ is just the set of functions in $C(G)$ that are uniformly continuous with respect to the left uniformity of $G$.

(iv) Most of the assertions here have easy proofs which we omit. That $\psi^{*}$ injects $L U C$ into $R U C$ when $X$ is compact follows from (i) and (ii) and the fact that $\psi^{*}$ maps $U_{L}$ onto $U_{R}$. [That $\psi^{*}(L U C) \backslash R U C$ can be nonvoid if $X$ is not compact is shown in the next remark.] A function in $R U C \backslash \psi^{*}(L U C)$ is constructed on the left-group $[0,1] \times R$ after Theorem 1.4.

REMARK. That $L U C \backslash U_{L}$ can be nonvoid if $X$ is not compact follows easily from the observation that $C(X)=L U C(X)$ for every topological left-zero semigroup $X$. Thus, for the left-group $R \times[0,1)$, where $R$, the set of real numbers, and $[0,1)=R / Z$ have their usual metric uniformities, the function $f$ defined by

$$
f(x, s)=\sin \left(x^{2}\right) \text { for all } x \in R, s \in[0,1)
$$

is in $L U C \backslash U_{L}$. A function in $L U C \backslash R U C$ on this same left-group is defined by

$$
f(x, s)=s(1-s) \sin x s \quad \text { for all } x \in R, s \in[0,1) \text {. }
$$

Obviously, $f \in L U C=\psi^{*}(L U C)$ [notation as in Theorem 1.1, (iv)] ; and $f \notin R U C$ by Theorem 1.1 , (iii).

It is known that $A P \subset L U C \cap R U C$ always and that

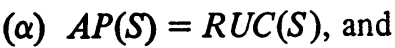

(B) $A P(S)=L U C(S)$

at least if $S$ is a compact semitopological semigroup [12, Lemma 4.11] or a topological left-zero semigroup. In view of the Ascoli-Arzelà theorem we have the following corollary to Theorem 1.1, (iii), which gives another noncompact setting in which the equality $(\alpha)$ holds. [In fact, the statements of Theorem 1.1 , (iii), and the present corollary make sense and remain true for semigroups of the form $S=X \times T$ where $X$ is a topological left-zero semigroup and $T$ is a compact semitopological semigroup.] Together with the second example of the immediately preceding remark, this corollary also shows that the equality $(\beta)$ can fail to hold in this setting.

COROLlary 1.2. Let $S=X \times G$ be a topological left-group with $G$ compact. Then $A P=R U C$.

Since bounded almost periodic functions depending uniformly on parameters are almost periodic functions on certain left-groups [see the Introduction], the next theorem can be viewed as a considerable generalization of Theorem 2.2, p. 52 , of [5]. 
THEOREM 1.3. Let $X \times G$ be a topological left-group with $X$ compact. Then $A P \subset U_{L} \cap U_{R}$.

Proof. Since $A P \subset L U C$ always and $L U C=U_{L}$ in this setting we need only show $A P \subset U_{R}$. Suppose $f \in A P$ and $\epsilon>0$ are given. Then $F: X \rightarrow$ $C(G)$, defined by

$$
(F(x))(s)=f(x, s)
$$

for all $x \in X, s \in G$, is uniformly continuous, hence there is a $U_{1} \in U$, the unique uniformity of $X$, such that

$$
\left\|F\left(x_{1}\right)-F\left(x_{2}\right)\right\|<\epsilon / 3
$$

whenever $\left(x_{1}, x_{2}\right) \in U_{1}$. Also, there is a $U \in U$ such that $U^{2} \subset U_{1}$ and there is a finite subset $\left\{x_{1}, x_{2}, \ldots, x_{n}\right\} \subset X$ such that

$$
\bigcup_{1}^{n}\left\{y \in X \mid\left(x_{i}, y\right) \in U\right\}=X .
$$

Let $V$ be a neighbourhood of $e \in G$ such that

$$
\left|f\left(x_{i}, s\right)-f\left(x_{i}, t\right)\right|<\epsilon / 3, \quad i=1,2, \ldots, n,
$$

whenever $s^{-1} t \in V$. So, given $\left(y_{1}, y_{2}\right) \in U$ and $s, t \in G$ satisfying $s^{-1} t \in V$, then $\left(x_{i}, y_{1}\right) \in U$ for some $i$ and $\left(x_{i}, y_{2}\right) \in U_{1}$, hence

$$
\begin{aligned}
\left|f\left(y_{1}, s\right)-f\left(y_{2}, t\right)\right| \leqslant & \left|f\left(y_{1}, s\right)-f\left(x_{i}, s\right)\right| \\
& +\left|f\left(x_{i}, s\right)-f\left(x_{i}, t\right)\right|+\left|f\left(x_{i}, t\right)-f\left(y_{2}, t\right)\right|<\epsilon .
\end{aligned}
$$

We are done.

REMARK. Since $C(X)=A P(X)$ for any topological left-zero semigroup $X$, the first example given in the remark following Theorem 1.1 shows that $A P \backslash\left(U_{L} \cup U_{R}\right)$ can be nonvoid if $X$ is not compact.

The last positive result of this section is a theorem about semitopological left-groups whose underlying topological spaces are of metric type and is a generalization of a theorem of Rao [15, Theorem 2]; the main idea of the proof is taken from Rao's proof.

THEOREM 1.4. Let $S=X \times G$ be a semitopological left-group where $X$ is a first countable topological space and $G$ is a complete metric space. Then $R U C=R M C$.

Proof. We assume $S$ is not discrete and must show $R M C \subset R U C$. We do this by showing $f \notin R M C$ if $f \notin R U C$. Suppose $f \notin R U C$. Then there exist 
$\epsilon>0,\left\{\left(x_{n}, t_{n}^{\prime}\right)\right\} \subset S,\left\{\left(y_{n}, s_{n}^{\prime}\right)\right\} \subset S$ and $\left(y^{\prime}, s\right) \in S$ such that $\lim _{n}\left(y_{n}, s_{n}^{\prime}\right)=$ $\left(y^{\prime}, s\right)$ and

$$
\left|f\left(\left(x_{n}, t_{n}^{\prime}\right)\left(y_{n}, s_{n}^{\prime}\right)\right)-f\left(\left(x_{n}, t_{n}^{\prime}\right)(y, s)\right)\right|=\left|f\left(x_{n}, t_{n}^{\prime} s_{n}^{\prime}\right)-f\left(x_{n}, t_{n}^{\prime} s\right)\right| \geqslant \epsilon
$$

for all $n$. We write $t_{n}^{\prime} s_{n}^{\prime}=t_{n}, t_{n}^{\prime} s=s_{n}$, have

$$
\lim _{n} s_{n}^{-1} t_{n}=e \in G
$$

and may assume:

(i) $\lim _{n} f\left(x_{n}, t_{n}\right)=0$,

(ii) $\lim _{n} f\left(x_{n}, s_{n}\right)=1$.

We now fix a point $y \in X$ and consider the sequence $\left\{L_{\left(x_{n}, s_{n}\right)} f\right\}$ of left translates of $f$. At least one of the following two situations must arise.

1. There is a sequence $\left\{\left(y, r_{k}\right)\right\} \subset S$ such that

$$
\lim _{k}\left(y, r_{k}\right)=(y, e)
$$

and a subsequence $\left\{L_{\left(x_{m}, s_{m}\right)} f\right\}$ of $\left\{L_{\left(x_{n}, s_{n}\right)} f\right\}$ such that

$$
\lim _{m} L_{\left(x_{m}, s_{m}\right)} f\left(y, r_{k}\right)=p_{k}
$$

with $\left|p_{k}\right| \leqslant 1 / 2$ for each $k$. If $\mu$ is a cluster point in $\beta S$ of the sequence of evaluation functionals on $C(S)$ corresponding to $\left\{\left(x_{n}, s_{n}\right)\right\}$, then

for all $k$, while

$$
\left|\mu\left(R_{\left(y, r_{k}\right)} f\right)\right|=\lim _{m}\left|L_{\left(x_{m}, s_{m}\right)} f\left(y, r_{k}\right)\right|=\left|p_{k}\right| \leqslant 1 / 2
$$

$$
\mu\left(R_{(y, e)} f\right)=\lim _{m} f\left(x_{m}, s_{m}\right)=1 .
$$

Since $\lim _{k}\left(y, r_{k}\right)=(y, e)$, this implies $f \notin R M C[12$, Theorem 3.1].

2. If $d$ denotes the metric in $G$, there is a disc

$$
D((y, e), \delta)=\{(y, u) \in S \mid d(u, e)<\delta\}
$$

with $\delta>0$ and a subsequence $\left\{L_{\left(x_{m}, s_{m}\right)} f\right\}$ of $\left\{L_{\left(x_{n}, s_{n}\right)} f\right\}$ such that

$$
\left|L_{\left(x_{m}, s_{m}\right)} f(y, t)\right| \leqslant 1 / 2
$$

for only finitely many $m$ for each $(y, t) \in D((y, e), \delta)$. We let $\xi$ be a cluster point in $\beta S$ of the sequence of evaluation functionals on $C(S)$ corresponding to $\left\{\left(x_{m}, t_{m}\right)\right\}$, hence 


$$
\xi(f)=\xi\left(R_{(y, e)} f\right)=0,
$$

and find a sequence $\left\{\left(y, r_{k}\right)\right\} \subset S$ such that $\lim _{k}\left(y, r_{k}\right)=(y, e)$, but $\left|\xi\left(R_{\left(y, r_{k}\right)} f\right)\right| \geqslant 1 / 2$ for all $k$. This will imply $f \notin R M C$.

For a fixed $k \geqslant 1$, we find $\left(y, r_{k}\right)$ as follows. Choose $\left(y, u_{k}\right) \in S$ such that $u_{k} \neq e$ and $d\left(u_{k}, e\right)<2^{-k} \delta$, let

$$
D_{k}=D\left(\left(y, u_{k}\right), d\left(u_{k}, e\right) / 2\right)^{-} \quad(\text { closure in } S),
$$

and let $E_{m}=\left\{(y, r) \in D_{k}|| L_{\left(x_{m}, s_{m}\right)} f(y, r) \mid \geqslant 1 / 2\right\}$. Since $D_{k} \subset D((y, e), \delta)$,

$$
D_{k}=\bigcup_{i \geqslant 1}\left(\bigcap_{m>i} E_{m}\right) \text {. }
$$

The Baire category theorem applied to $D_{k}$ asserts the existence of an $i_{0}$ such that $\bigcap_{m \geqslant i_{0}} E_{m}$ has nonvoid interior $E^{0}$ in $D_{k}$. Thus, there is a $\left(y, r_{k}\right) \in E^{0}$ and a $\delta_{k}>0$ such that $D\left(\left(y, r_{k}\right), \delta_{k}\right) \subset E^{0}$. Explicitly, for all $(y, r) \in D\left(\left(y, r_{k}\right), \delta_{k}\right)$ and for all $m \geqslant i_{0}$

$$
\left|L_{\left(x_{m}, s_{m}\right)} f(y, r)\right| \geqslant 1 / 2 .
$$

We now observe that, for a given $\epsilon>0$,

$$
\left|\xi\left(R_{\left(y, r_{k}\right)} f\right)-R_{\left(y, r_{k}\right)} f\left(x_{m}, t_{m}\right)\right|<\epsilon
$$

for infinitely many $m$, that

$$
\left(x_{m}, s_{m}\right)\left(y, s_{m}^{-1}\right)=\left(x_{m}, e\right)
$$

for all $m$, that

$$
\lim _{m}\left(y, s_{m}^{-1}\right)\left(x_{m}, t_{m}\right)\left(y, r_{k}\right)=\left(y, r_{k}\right)
$$

and hence that

$$
\left|R_{\left(y, r_{k}\right)} f\left(x_{m}, t_{m}\right)\right|=\left|L_{\left(x_{m}, s_{m}\right)}\left(\left(y, s_{m}^{-1}\right)\left(x_{m}, t_{m}\right)\left(y, r_{k}\right)\right)\right| \geqslant 1 / 2
$$

for all large $m$, and have $\left|\xi\left(R_{\left(y, r_{k}\right)} f\right)\right| \geqslant 1 / 2$. The proof is complete.

The left-group $S=[0,1] \times R$. [Here $[0,1]$ and the real numbers $R$ have their usual metric and topology. Also $U_{L}=U_{R}$ since $R$ is abelian.] Consider the function $f \in C(S)$ defined as follows: for each natural number $n$, put 
$(*)$

$$
\begin{aligned}
& h_{n}(x)= \begin{cases}2^{n+2} x-2, & 2^{-n-1} \leqslant x \leqslant 3\left(2^{-n-2}\right), \\
-2^{n+2} x+4, & 3\left(2^{-n-2}\right) \leqslant x \leqslant 2^{-n}, \\
0, & \text { otherwise, }\end{cases} \\
& f_{n}(s)= \begin{cases}(s-n)(n+1-s), & n \leqslant s \leqslant n+1, \\
0, & \text { otherwise, }\end{cases}
\end{aligned}
$$

and define $f(x, s)=\Sigma_{n} h_{n}(x) f_{n}(s)$.

It is easy to check that $f \notin U_{R}=U_{L}=L U C$. However, since $\left|h_{n}(x)\right| \leqslant 1 / 4$ for all $x \in[0,1]$ and for all $n$ and the functions $\left\{f_{n}\right\}$ are uniformly equicontinuous on $R$ [ $\delta=\epsilon$ will do], Theorem 1.1, (iii) implies $f \in R U C$.

We next prove that $f \in W A P$. Let $\left\{\left(y_{i}, t_{i}\right)\right\}$ be a sequence in $S$; we must find a weakly convergent subsequence of $\left\{L_{\left(y_{i}, t_{i}\right)} f\right\}$. By assuming [as we may] that

$$
\lim _{i} y_{i}=y_{0} \in[0,1]
$$

and that $\lim _{i} t_{i}=t_{0} \in R$ or $\lim _{i}\left|t_{i}\right|=\infty$, and dealing with some easy cases (using, where necessary the facts that $\left\{L_{\left(y_{i}, t_{i}\right)} f\right\} \subset C_{00}(S)$, the subset of $C(S)$ whose members have compact support, and that, in bounded subsets of $C_{00}(S)$, uniform convergence on compact subsets of $S$ implies weak convergence), we are left with the only difficult case, the one where $y_{0}=0$ and $\lim _{i}\left|t_{i}\right|=\infty$.

In this case, for all large enough $i$ we may assume

$$
2^{-n_{i}-1}<y_{i} \leqslant 2^{-n_{i}}
$$

for some natural number $n_{i}$. Also we may assume that the sequence

$$
\left\{\left(2^{-n_{i}}-y_{i}\right) 2^{n_{i}-1}\right\}
$$

converges to $y^{\prime}, 0 \leqslant y^{\prime} \leqslant 1 / 4$, and that

$$
\lim _{i}\left(n_{i}-t_{i}\right)=t^{\prime} \in R \text { or } \lim _{i}\left|n_{i}-t_{i}\right|=\infty .
$$

If $\lim _{i}\left|n_{i}-t_{i}\right|=\infty,\left\{L_{\left(y_{i}, t_{i}\right)} f\right\}$ converges to $0 \in C_{00}(S)$ uniformly on compact subsets of $S$, hence weakly. If $\lim _{i}\left(n_{i}-t_{i}\right)=t^{\prime}$, then

$$
\lim _{i}\left\|L_{\left(y_{i}, t_{i}\right)} f-g\right\|=0,
$$

where $g \in C(S)$ is defined by

$$
g(x, s)=\left\{\begin{array}{l}
h_{1}\left(2^{-1}-y^{\prime}\right)\left(s-t^{\prime}\right)\left(t^{\prime}+1-s\right), t^{\prime} \leqslant s \leqslant t^{\prime}+1, \\
0, \text { otherwise. }
\end{array}\right.
$$

[ $h_{1}$ is defined by (*) above.] Thus $f \in W A P$ as asserted. 
Remarks about the function $f$. (a) The production of this function completes the proof of Theorem 1.1, (ii). It also completes the proof of Theorem 1.1, (iv), namely, it shows $R U C \backslash \psi^{*}(L U C)$ can be nonvoid. [To see the truth of the second assertion, one can use the fact that $\psi^{*}(f) \in W A P \subset R M C=R U C$, which follows from another part of Theorem 1.1, (iv) and Theorem 1.4.]

(b) For a locally compact left-group $S=X \times G$ with $X$ compact, (A) of the Introduction holds as always. For the particular case $S=[0,1] \times R$, we summarize the established breakdown of (B) of the Introduction.

$$
W A P \not \subset L U C, \quad L U C \not \subset W A P, \quad L U C \neq L M C, \quad U_{R} \neq R U C .
$$

However, we do still have $L U C=U_{L}$ [Theorem 1.1, (i)], $A P \subset U_{L} \cap U_{R}$ [Theorem 1.3], and $W A P \subset R U C$ [Theorem 1.4]. [The proof that $L U C \not \subset W A P$ can be achieved by extending a function $f \in L U C(R) \backslash W A P(R)$, such as $f(s)=$ $\arctan s$, to a function $F$ on $S$ by means of the formula $F(x, s)=f(s)$. Obviously, such an $F$ is in $L U C \backslash W A P=U_{R} \backslash W A P$.]

(c) In the next section the function $f$ will be used to show that the weakly almost periodic compactification of $[0,1] \times R$ is not the product of $[0,1]$ and the weakly almost periodic compactification of $R$.

2. Compactifications of products. In [7, Corollary 4.4] deLeeuw and Glicksberg proved that the almost periodic compactification of a product of abelian topological semigroups with identity is the product of the almost periodic compactifications of the component semigroups. We show here that this conclusion remains true for a product of semitopological semigroups, one of which has a left identity, the other having a right identity. [Thus the result holds for left-groups.] We also give an example to illustrate the necessity of the hypotheses concerning the identities and then turn to the analogous assertion for the weakly almost periodic compactification which can be false even in familiar settings, but is true at least if one member of the product is a compact group. A positive result is established for one further compactification, that associated with the $C^{*}$-algebra $R M C$.

We first establish some notation. Let $S$ be a semitopological semigroup. We regard the almost periodic [respectively, weakly almost periodic] compactification aS [respectively, $w S$ ] of $S$ as a compact topological [respectively, semitopological] semigroup together with a continuous homomorphism $\alpha$ [respectively, $\omega$ ] of $S$ onto a dense subset of $a S$ [respectively, $w S$ ], for which the following universal mapping property holds:

if $\gamma$ is a continuous homomorphism of $S$ into a compact topological [respectively, semitopological] semigroup $T$, then there is a unique 
continuous homomorphism $\gamma_{1}$ of $a S$ [respectively, $w S$ ] into $T$ such that

for all $s \in S$.

$$
\begin{aligned}
& \left(\gamma_{1} \circ \alpha\right)(s)=\gamma(s) \\
& \text { [respectively, } \left.\left(\gamma_{1} \circ \omega\right)(s)=\gamma(s)\right]
\end{aligned}
$$

A useful property of the adjoint map $\alpha^{*}$ [respectively, $\omega^{*}$ ] is that it is an isometric isomorphism of $C(a S)$ [respectively, $C(w S)$ ] onto $A P(S)$ [respectively, $W A P(S)]$. See [3] for all these matters.

The next two lemmas are our basic techniques of proof in this section. They could be formulated in more general settings.

LEMmA 2.1. Let $S$ be a semitopological semigroup with subsets $H$ and $K$ such that $S=H K$ and let $(\delta, d S)$ be either $(\alpha, a S)$ or $(\omega, w S)$. Suppose the action

$$
(\delta H)^{-} \times(\delta K)^{-} \rightarrow d S \quad[\text { closure in } d S]
$$

is jointly continuous. Then $d S=(\delta H)^{-}(\delta K)^{-}$.

Proof. The desired conclusion is an immediate consequence of the facts that $(\delta H)^{-}(\delta K)^{-}$contains $\delta S$ which is dense in $d S$ and that the continuous image of a compact set is compact.

LEMMA 2.2. Suppose $T_{1}$ and $T_{2}$ are semitopological semigroups, $T_{1}$ having a right identity $e_{1}$ and $T_{2}$ having a left identity $e_{2}$. If $S=T_{1} \times T_{2}$, then

and

$$
W A P\left(T_{1}\right)=\left.W A P(S)\right|_{T_{1} \times\left\{e_{2}\right\}}
$$

$$
W A P\left(T_{2}\right)=\left.W A P(S)\right|_{\left\{e_{1}\right\} \times T_{2}} .
$$

Similar statements hold for $A P\left(T_{1}\right)$ and $A P\left(T_{2}\right)$.

Proof. The projections

$$
\begin{aligned}
& \left(t_{1}, t_{2}\right) \rightarrow\left(t_{1}, e_{2}\right): S \rightarrow T_{1} \times\left\{e_{2}\right\}, \\
& \left(t_{1}, t_{2}\right) \rightarrow\left(e_{1}, t_{2}\right): S \rightarrow\left\{e_{1}\right\} \times T_{2}
\end{aligned}
$$

are retractions in the category of semitopological semigroups. The result follows from [2, Lemma 2].

THEOREM 2.3. If $S=T_{1} \times T_{2}$, where $T_{1}$ and $T_{2}$ are semitopological semigroups and $T_{1}$ has a right identity $e_{1}$ while $T_{2}$ has a left identity $e_{2}$, then $a S=a T_{1} \times a T_{2}$. 
Proof. We have $S=\left(T_{1} \times\left\{e_{2}\right\}\right)\left(\left\{e_{1}\right\} \times T_{2}\right)$. Since $a S$ is always a topological semigroup, Lemma 2.1 implies

$$
a S=\alpha\left(T_{1} \times\left\{e_{2}\right\}\right)^{-} \alpha\left(\left\{e_{1}\right\} \times T_{2}\right)^{-} .
$$

[Here $\alpha$ is the continuous homomorphism of $S$ into $a S$.] But we may identify $\alpha\left(T_{1} \times\left\{e_{2}\right\}\right)^{-}$with $a T_{1}$ and $\alpha\left(\left\{e_{1}\right\} \times T_{2}\right)^{-}$with $a T_{2}$, by Lemma 2.2. It remains to show that the equality $\tau_{1} \sigma_{1}=\tau_{2} \sigma_{2}$ with $\tau_{1}, \tau_{2} \in a T_{1}, \sigma_{1}, \sigma_{2} \in a T_{2}$, implies $\tau_{1}=\tau_{2}$ and $\sigma_{1}=\sigma_{2}$. Suppose, for example, that $\tau_{1} \neq \tau_{2}$. Then there is a function $f \in C\left(a T_{1}\right)$ with $f\left(\tau_{1}\right)=1, f\left(\tau_{2}\right)=0$. The image of $f$ in $A P\left(T_{1}\right)$ under the adjoint of the map from $T_{1}$ into $a T_{1}$ can be extended to a function $g \in$ $A P(S)$ which satisfies $g(t, s)=g\left(t, e_{2}\right)$ for all $t \in T_{1}, s \in T_{2}$. But then this $g$ is the image under $\alpha^{*}$ of a function $h \in C(a S)$ which satisfies

$$
h(\tau \sigma)=h(\tau)=f(\tau)
$$

for all $\tau \in a T_{1}, \sigma \in a T_{2}$; in particular

$$
1=f\left(\tau_{1}\right)=h\left(\tau_{1} \sigma_{1}\right)=h\left(\tau_{2} \sigma_{2}\right)=f\left(\tau_{2}\right)=0
$$

Thus $\tau_{1}=\tau_{2}$. That $\sigma_{1}=\sigma_{2}$ can be proved similarly.

Theorem 2.3 implies, in particular, that

$$
a(X \times G)=a X \times a G=\beta X \times a G
$$

for left-groups $X \times G$, a result that is implicit in [1].

An example is now given which shows the necessity of the hypotheses concerning the identities.

EXAMPLE. Let $[0,1]$ and the real numbers $R$ each be given the left-zero multiplication $x_{1} x_{2}=x_{1}$. Then $S=[0,1] \times R$ is also a left-zero semigroup and the function $f \in C(S)$ defined by $f(x, y)=\sin x y$ [where $x y$ is the ordinary product of real numbers] is in $A P(S)$ since $A P(S)=C(S)$ for left-zero semigroups. The existence of such an $f \in A P(S)$ implies $a S \neq a[0,1] \times a R$. [See Remark (iii) following Theorem 2.5 ahead.]

The only way in which the proof of the following theorem need differ from that of Theorem 2.3 is in the use of Ellis' theorem [9] to assert the joint continuity of the action $w S \times \omega(G) \rightarrow w S$.

THEOREM 2.4. If $S=T \times G$ where $G$ is a compact topological group and $T$ is a semitopological semigroup with right identity, then $w S=w T \times G$.

The next theorem gives a criterion for determining when a compactification of a product semigroup is the product of the corresponding compactifications of the component semigroups. The theorem is a generalization of part of a theorem 
of Ptak [14, (6.2)] and could be used to prove Theorems 2.3 and 2.4; in fact, deLeeuw and Glicksberg proved Corollary 4.4 of [7] using some of the ideas involved in this theorem and I. Glicksberg has pointed out to us that much of Theorem 2.4 can be proved in this way as well. As with Lemmas 2.1 and 2.2, Theorem 2.5 could be formulated in a more general setting.

THEOREM 2.5. Let $S=T_{1} \times T_{2}$ be a product of semitopological semigroups where $T_{1}$ has a right identity $e_{1}$ and $T_{2}$ has a left identity $e_{2}$. For $f \in$ $C(S)$, define $f_{t} \in C\left(T_{2}\right)$ and $f^{s} \in C\left(T_{1}\right)$ by

$$
f_{t}(s)=f^{s}(t)=f(t, s)
$$

for $t \in T_{1}, s \in T_{2}$ and put

$$
A_{f}=\left\{f_{t} \mid t \in T_{1}\right\}, \quad B_{f}=\left\{f^{s} \mid s \in T_{2}\right\} .
$$

Then $A_{f} \subset W A P\left(T_{2}\right)$ and $B_{f} \subset W A P\left(T_{1}\right)$ for every $f \in W A P(S)$. Also $w S=w T_{1}$ $\times w T_{2}$ if and only if one of $A_{f}$ or $B_{f}$ is relatively compact (in the norm topology) for every $f \in W A P(S)$. Thus both $A_{f}$ and $B_{f}$ are relatively compact for every $f \in W A P(S)$ if $w S=w T_{1} \times w T_{2}$. Similar statements hold for the almost periodic compactification and the RMC-compactification (which is discussed later in this section).

Proof. Let $f \in W A P(S)$. On account of the equalities

$$
R_{s_{1}} f_{t}(s)=R_{\left(e_{1}, s_{1}\right)} f(t, s) \text { and } L_{t_{1}} f^{s}(t)=L_{\left(t_{1}, e_{2}\right)} f(t, s) \text {, }
$$

we see that the right orbit of $f_{t}$ in $C\left(T_{2}\right)$ [respectively, left orbit of $f^{s}$ in $C\left(T_{1}\right)$ ] is a subset of the restriction to $\{t\} \times T_{2}$ [respectively, to $T_{1} \times\{s\}$ ] of the right orbit [respectively, left orbit] of $f$ in $C(S)$. Since restriction maps are norm, hence weakly, continuous, $A_{f} \subset W A P\left(T_{2}\right)$ and $B_{f} \subset W A P\left(T_{1}\right)$.

Suppose $w S=w T_{1} \times w T_{2}$. We wish to show, for example, that $B_{f}$ is relatively compact in $W A P\left(T_{1}\right)$. [Note that this is the same as showing $B_{f}$ is relatively compact in $C\left(T_{1}\right)$.] To do this, we put

$$
B_{f}^{\prime}=\left\{\hat{f}^{\sigma} \in C\left(w T_{1}\right) \mid \sigma \in w T_{2}\right\},
$$

where $\hat{f} \in C\left(w T_{1} \times w T_{2}\right)$ satisfies $\omega^{*} \hat{f}=f$ and $\hat{f}^{\sigma}(\tau)=\hat{f}(\tau, \sigma)$ for $\tau \in w T_{1}, \sigma$ $\in w T_{2} . B_{f}^{\prime}$ is compact, being the image under the continuous function $\sigma \rightarrow \hat{f}^{\sigma}$ of the compact set $w T_{2}$. The continuity of this function follows from an elementary property of the product topology and the compactness of $w T_{1}$. Since $B_{f} \subset \omega^{*} B_{f}^{\prime}$, we have reached the desired conclusion.

Conversely, suppose, for example, that $B_{f}$ is relatively compact for a given $f \in W A P(S)$. Suppose, as well, that $\tau \in w T_{1}$ and $\sigma \in w T_{2}$, and $\left\{t_{\gamma}\right\} \subset T_{1}$ and 
$\left\{s_{\delta}\right\} \subset T_{2}$ are nets such that

$$
\lim _{\gamma} g\left(t_{\gamma}\right)=\hat{g}(\tau) \text { and } \lim _{\delta} h\left(s_{\delta}\right)=\hat{h}(\sigma)
$$

for all $g \in W A P\left(T_{1}\right)$ and $h \in W A P\left(T_{2}\right)$, respectively. [Here, for example, $\hat{g} \in$ $C\left(w T_{1}\right)$ is such that $\omega^{*} \hat{g}=g$.] The relative compactness of $B_{f}$ tells us that a subnet of $\left\{f^{s_{\delta}}\right\}$ converges uniformly on $T_{1}$ to a function $g \in W A P\left(T_{1}\right)$. Since $f_{t} \in W A P\left(T_{2}\right)$ for all $t \in T_{1}$,

$$
\lim _{\delta} f^{s \delta}(t)=\lim _{\delta} f_{t}\left(s_{\delta}\right)=\left(f_{t}\right) \hat{(\sigma)}
$$

hence the function $g$ is uniquely determined and

$$
\lim _{\delta}\left\|f^{s} \delta-g\right\|=0
$$

Since $g \in W A P\left(T_{1}\right)$, we have the joint limit

$$
\lim _{\boldsymbol{\gamma}, \delta}\left|f\left(t_{\gamma}, s_{\delta}\right)-g(\tau)\right| \leqslant \lim _{\gamma, \delta}\left(\left|f^{s \delta}\left(t_{\gamma}\right)-g\left(t_{\gamma}\right)\right|+\left|g\left(t_{\gamma}\right)-\hat{g}(\tau)\right|\right)=0 .
$$

Hence, if $\kappa: C\left(w T_{1} \times w T_{2}\right) \rightarrow C(S)$ is defined by

$$
\kappa F(t, s)=F(\omega t, \omega s),
$$

it follows that $\kappa\left(C\left(w T_{1} \times w T_{2}\right)\right) \supset W A P(S)$; and it is easy to see the reverse inclusion. So $w S=w T_{1} \times w T_{2}$.

REMARKS. (i) The existence of the weakly almost periodic function $f$ that was constructed on the left-group $[0,1] \times R$ in $\$ 1$ implies that $w([0,1] \times R) \neq[0,1] \times w R$ since $B_{f} \subset C([0,1])$ is not equicontinuous and hence is not relatively compact.

(ii) In [7] it is asserted that $w\left(T_{1} \times T_{2}\right)$ is not always equal to $w T_{1} \times$ $w T_{2}$, where $T_{1}$ and $T_{2}$ are abelian topological semigroups with identity. I.

Glicksberg has informed us that the example that was intended is $R \times R$. The product of the integers $Z \times Z$ is another example and can be quickly handled. Using Grothendieck's criterion [10, Proposition 7], one can easily see that the function

$$
f(m, n)= \begin{cases}1, & \text { if } m=n \\ 0, & \text { if } m \neq n\end{cases}
$$

is in $W A P(Z \times Z)$, while $B_{f}$ is not relatively compact. Thus $w(Z \times Z) \neq w Z \times$ $w Z$.

(iii) The function $f$ defined on the left-zero semigroup $[0,1] \times R$ following 
Theorem 2.3 clearly has $B_{f}$ not relatively compact and, although this product semigroup does not satisfy the hypotheses concerning the identities of Theorem 2.5 , one can still use the argument of the second paragraph of the proof of that theorem, arguing by contradiction, to conclude that

$$
a([0,1] \times R) \neq[0,1] \times a R .
$$

(iv) The first conclusion of Theorem 2.5 can fail if one of the component semigroups is allowed to have no identity of any kind. For example, if $S=$ $[0,1] \times R$ has the multiplication

$$
(x, s)(y, t)=(0, s+t)
$$

then the function $f$ defined on $S$ by $f(x, s)=\sin x s$ is in $A P(S)$ while neither $A_{f}$ nor $B_{f}$ is relatively compact.

Our methods in this section can be applied to get a positive result about another compactification of a semitopological semigroup $S$, that corresponding to $R M C(S)$. We regard this compactification $m S$ as a compact semigroup together with a continuous homomorphism $\mu$ of $S$ onto a dense subset of $m S$ : in $m S$ the maps

$$
x \rightarrow y x \text { and } x \rightarrow x \mu(s)
$$

from $m S$ are continuous at least for all $y \in m S$ and for all $s \in S$ and a universal mapping property is satisfied [12, Theorem 3.4]. Namely, if $\boldsymbol{\gamma}$ is a continuous homomorphism of $S$ into a compact semigroup $T$ with continuity properties analogous to (*), then there is a unique continuous homomorphism $\gamma_{1}$ of $m S$ into $T$ such that

$$
\left(\gamma_{1} \circ \mu\right)(s)=\gamma(s)
$$

for all $s$ in $S$.

THEOREM 2.6. If $S=T \times G$ where $G$ is a compact topological group and $T$ is a semitopological semigroup with right identity, then $m S=m T \times G$.

Proof. The conclusion of Lemma 2.1 also holds if $(\delta, d S)=(\mu, m S)$ and we can use Ellis' theorem, as in the proof of Theorem 2.4, to assert the joint continuity of the action

$$
m S \times \mu(G) \rightarrow m S
$$

The rest of the proof is like that of Theorem 2.3.

REMARKS. (i) The existence of the function in $L U C \backslash R U C \subset L M C$ on the left-group $R \times[0,1)$ constructed in the Remark following Theorem 1.1 shows 
that an $L M C$ analogue of Theorem 2.6 does not exist. It also shows that the hypothesis that $T$ have a right identity is essential for the conclusion of Theorem 2.6 , i.e., if $S$ is the topological right-group

$$
R \times[0,1) \quad[(x, s)(y, t)=(y,(s+t)(\bmod 1))],
$$

then $m S \neq m R \times m[0,1)=m R \times[0,1)$.

(ii) In the special case that the extra hypotheses that $T$ is a left-group and $T$ and $G$ are first countable are made, the conclusion of Theorem 2.6 can also be proved using Corollary 1.2 and Theorems 1.4 and 2.3. In this case we have

$$
m S=m T \times G=a T \times G .
$$

3. Analytic weakly almost periodic functions. Certain classical questions about almost periodic functions of a real variable and analytic almost periodic functions are considered in this section. An "analytic almost periodic" function is not defined to be an entire function which is almost periodic on the topological group $C$ for the obvious reason that the only such functions are constants. Bohr defined analytic almost periodic functions to be almost periodic functions depending on a real parameter which are analytic on a strip (or half-plane). We are led to an obvious generalization. For notation, let

$$
-\infty \leqslant a<c \leqslant d<b \leqslant+\infty,
$$

and let

$$
(a, b)=\{z \in C: a<\operatorname{Re} z<b\}
$$

and

$$
[c, d]=\{z \in C: c \leqslant \operatorname{Re} z \leqslant d\}
$$

Let $A(a, b)$ be the space of analytic functions on $(a, b)$, and let $A^{*}(a, b)$ denote the subspace consisting of those functions $f \in A(a, b)$ with $\left.f\right|_{[c, d]}$ bounded for every closed strip $[c, d] \subset(a, b)$. Consider $[c, d] \subset R^{2}$, and let $S_{[c, d]}$ denote the locally compact topological left-group $X \times G$, where $X$ is the real interval $[c, d]$ and $G$ is the additive group of real numbers.

Definition. Let $f \in A^{*}(a, b)$. If $f \in W A P\left(S_{[c, d]}\right)$ [respectively, $f \in$ $\left.A P\left(S_{[c, d]}\right)\right]$ for every closed strip $[c, d] \subset(a, b)$ then $f$ is called analytic weakly almost periodic [respectively, analytic almost periodic] on $(a, b)$.

Parallel definitions for the spaces $L M C, L U C, R M C, R U C$, and $U_{L}=U_{R}$ are possible, of course, but the next theorem shows that we would get $A^{*}(a, b)$ in each such case. The example following this theorem shows that the subspace of analytic weakly almost periodic functions is proper in $A^{*}(a, b)$. 
THEOREM 3.1. Let $f \in A^{*}(a, b)$. Then $f$ and all its derivatives are uniformly continuous on every closed strip $[c, d] \subset(a, b)$.

Proof [5, Theorem 3.7, p. 72].

EXAmple. The function $f$ defined on the strip $(0,1)$ by

$$
f(z)=\sum_{n=1}^{\infty}(n i-z)^{-2}
$$

is a member of $A^{*}(a, b)$, but $g \in C(R)$ defined by

$$
g(s)=f\left(x_{0}+i s\right)
$$

is not in $W A P(R)$ for any $x_{0} \in(0,1)$. Thus $f$ is not analytic weakly almost periodic. (See Theorem 3.5 ahead in this regard.)

We will consider analogues of the following well-known classical theorems:

A. The derivative of an almost periodic function of a real variable is almost periodic provided that it is uniformly continuous.

B. An antiderivative of an almost periodic function of a real variable is almost periodic provided that it is bounded.

C. If the restriction of $f \in A^{*}(a, b)$ to any single vertical line is an almost periodic function of a real variable, then $f$ and all its derivatives are analytic almost periodic on $(a, b)$.

Theorem B is the basis for the vast literature on almost periodic solutions to ordinary differential equations. We show by example (following Theorem 3.3) that, unfortunately, the analogue for weakly almost periodic functions of Theorem B is not true; and, therefore, the development of a theory of weakly almost periodic solutions to differential equations cannot be so fruitful. The analogues of $\mathrm{A}$ and $\mathrm{C}$ are given in Theorems 3.3 and 3.5 and Corollary 3.7 below.

LEMMA 3.2. Let $G$ be a locally compact group. If $f \in W A P(G)$ and $g \in$ $L^{1}(G)$, then the convolution $f * g$ is weakly almost periodic.

Proof. Let $\mu$ be left Haar measure on $G$. Note that if $\lambda$ is a continuous linear functional on $W A P(G)$, then the function

$$
s \rightarrow \lambda\left(L_{s} f\right)
$$

is weakly almost periodic [6, Lemma 5.13]. Whence, since

$$
f * g(s)=\int L_{s} f(t) g\left(t^{-1}\right) d \mu(t),
$$

we have that $f * g \in W A P(G)$. 
THEOREM 3.3. Suppose that $f \in W A P(R)$ and that $f$ is everywhere differentiable. Then the derivative $f^{\prime}$ is weakly almost periodic if and only if $f^{\prime}$ is uniformly continuous.

Proof. That weakly almost periodic functions on locally compact groups are uniformly continuous is well known. Suppose, now, that $f^{\prime}$ is uniformly continuous. Then, given $\epsilon>0$, we can choose a continuously differentiable function $\psi$ with compact support so that

$$
\left\|f^{\prime} * \psi-f^{\prime}\right\|_{\infty}<\epsilon .
$$

Integrating by parts and using the fact that $\psi$ has compact support, we get that

$$
\begin{aligned}
f^{\prime} * \psi(x) & =\int f^{\prime}(x-y) \psi(y) d y \\
& =\int f(x-y) \psi^{\prime}(y) d y=f * \psi^{\prime}(x) .
\end{aligned}
$$

But since $\psi$ has compact support, so does $\psi^{\prime}$. Thus, by Lemma 3.2, we have that $f^{\prime} * \psi$ is weakly almost periodic. But then, $f^{\prime}$ may be uniformly approximated by weakly almost periodic functions; since $W A P(R)$ is norm closed in $C(R)$, we conclude that $f^{\prime} \in W A P(R)$.

EXAMPLE. Let $f$ be the inverse trigonometric function

$$
f(x)=\arctan x .
$$

Then, since the derivative $f^{\prime}$ vanishes at infinity, the derivative of $f$ is weakly almost periodic. However, $f$ is not weakly almost periodic even though it is bounded (cf., statement B). To see that $f$ is not weakly almost periodic, take any sequence $n \rightarrow x_{n}$ in $R$ which goes to $+\infty$; take, say, $x_{n}=n$. Then, since $\left\{L_{n}(f)\right\}$ converges pointwise on $R$ to the constant function $\pi / 2$, we have that $\pi / 2$ is the only possible weak cluster point of $\left\{L_{n}(f)\right\}$. However, weak convergence on $R$ is the same as pointwise convergence on the Stone-Čech compactification of $R$, and the constant function $\pi / 2$ is not a pointwise cluster point of the sequence of extended functions.

For Theorem 3.5, we will need the following corollary to the Vitali Convergence Theorem:

THEOREM 3.4. Let $\left\{f_{n}\right\}$ be a uniformly bounded sequence of functions in $A(c, d)$. Then there is a subsequence of $\left\{f_{n}\right\}$ which converges uniformly on compact subsets to a limit $F$; the limit being, therefore, analytic in $(c, d)$.

Proof [16, Corollary 5.22, p. 169].

TheOREM 3.5. Let $f \in A^{*}(a, b)$. Suppose that, for some $x_{0}$ with $a<x_{0}$ $<b$, the function $g$ defined by 


$$
g(t)=f\left(x_{0}+i t\right), \quad t \in R,
$$

is in $W A P(R)$. Then $f$ is analytic weakly almost periodic on $(a, b)$.

Proof. Let $\left\{a_{n}\right\}$ be a sequence of real numbers, and let $z_{n}=x_{0}+i a_{n}$. Considering the strip $(a, b)$ to be a left-group, then

$$
R_{z_{n}} f(s+i t)=f\left(s+i\left(t+a_{n}\right)\right) .
$$

By Theorem 3.4 we may assume, by passing to a subsequence if necessary, that the sequence of analytic functions $\left\{R_{z_{n}} f\right\}$ converges uniformly on compact subsets to a function $F \in A^{*}(a, b)$. Define a function $G$ on $R$ by

$$
G(t)=F\left(x_{0}+i t\right) \text {. }
$$

Since $R_{a_{n}} g=\left.R_{z_{n}} f\right|_{x_{0}+i R}$, we have that $G$ is the only pointwise cluster point of the sequence $\left\{R_{a_{n}} g\right\}$. Consequently, since $g \in W A P(R)$, we must have that $\left\{R_{a_{n}} g\right\}$ converges weakly to $G$.

We now want to show that $\left\{R_{z_{n}} f\right\}$ converges weakly to $F$ on every closed strip $[c, d] \subset(a, b)$, with $c \leqslant x_{0} \leqslant d$. It suffices to show that $\left\{R_{z_{n}} f\right\}$ converges quasi-uniformly to $F$, since a sequence of bounded continuous functions $\left\{g_{n}\right\}$ converges weakly to a continuous function $G$ if and only if it is uniformly bounded and, together with every subsequence, converges to $G$ quasi-uniformly [8, Lemma 30, p. 281$]$. (A sequence $\left\{g_{n}\right\}$ converges quasi-uniformly to a function $G$ if it converges pointwise to $G$ and if, given $\epsilon>0$ and index $N$, there are indices $n_{k} \geqslant N, k=1,2, \ldots, m$, such that

$$
\min _{1 \leqslant k \leqslant m}\left|g_{n_{k}}(t)-G(t)\right|<\epsilon
$$

for every $t$.)

Let $\epsilon>0$. Since $f$ and $F$ are both uniformly continuous on $[c, d]$, there is a $\delta>0$ such that if $\left|x_{1}-x_{2}\right|<\delta$, then

$$
\left|f\left(x_{1}+i t\right)-f\left(x_{2}+i t\right)\right|<\epsilon / 3
$$

and

$$
\left|F\left(x_{1}+i t\right)-F\left(x_{2}+i t\right)\right|<\epsilon / 3
$$

for every $t \in R$. Also, given an index $N$, there are indices $n_{k} \geqslant N, k=1,2$, $\ldots, m$, such that

$$
\min _{1 \leqslant k \leqslant m}\left|g\left(t+a_{n_{k}}\right)-G(t)\right|<\epsilon / 3
$$


for every $t \in R$; which is to say,

$$
\min _{1 \leqslant k<m}\left|f\left(x_{0}+i\left(t+a_{n_{k}}\right)\right)-F\left(x_{0}+i t\right)\right|<\epsilon / 3
$$

for every $t \in R$. Thus, for any $x$ with $c \leqslant x \leqslant d$ and $\left|x-x_{0}\right|<\delta$, we get

$$
\begin{aligned}
\mid f(x+i(t+ & \left.\left.a_{n_{k}}\right)\right)-F(x+i t) \mid \\
\leqslant & \left|f\left(x+i\left(t+a_{n_{k}}\right)\right)-f\left(x_{0}+i\left(t+a_{n_{k}}\right)\right)\right| \\
& +\left|f\left(x_{0}+i\left(t+a_{n_{k}}\right)\right)-F\left(x_{0}+i t\right)\right| \\
& +\left|F\left(x_{0}+i t\right)-F(x+i t)\right|
\end{aligned}
$$

and the least of these is less than $\epsilon$. Whence, $\left\{R_{z_{n}} f\right\}$ converges quasi-uniformly to $F$ on the strip $\left(x_{0}-\delta, x_{0}+\delta\right) \cap[c, d]$. Since $\delta$ did not depend on $x_{0}$, we may choose another point and repeat; we conclude that $\left\{R_{z_{n}} f\right\}$ converges quasiuniformly to $F$ on all of the strip $[c, d]$, as desired.

CoROllaRY 3.6 (CF. [5, THEOREM 3.12]). If $\left\{f_{n}\right\}$ is a sequence of analytic weakly almost periodic functions on a strip $(a, b)$ which converges uniformly on every closed strip $[c, d] \subset(a, b)$ to a function $f$, then $f$ is analytic weakly almost periodic on $(a, b)$.

COROLlaRY 3.7. If the function $f$ is analytic weakly almost periodic on the strip $(a, b)$, then all its derivatives are analytic weakly almost periodic in $(a, b)$.

Proof. 3.1, 3.3, and 3.5.

\section{REFERENCES}

1. R. P. Hunter and L. W. Anderson, On the infinite sub-semigroups of a compact semigroup, Fund. Math. 74 (1972), 1-19. MR 45 \#5265.

2. J. F. Berglund, On extending almost periodic functions, Pacific J. Math. 33 (1970), $281-289$.

3. J. F. Berglund and K. H. Hofmann, Compact semitopological semigroups and weakly almost periodic functions, Lecture Notes in Math., no. 42, Springer-Verlag, Berlin, 1967.

4. N. Bourbaki, Topologie générale, Hermann, Paris, 1971.

5. C. Corduneanu, Almost periodic functions, Interscience, New York, 1968.

6. K. deLeeuw and I. Glicksberg, Applications of almost periodic compactifications, Acta Math. 105 (1961), 63-97. MR 24 \#A1632.

7. Almost periodic functions on semigroups, Acta Math. 105 (1961), 99-140. MR 24 \#A 1633.

8. N. Dunford and J. T. Schwartz, Linear operators. I: General theory, Pure and Appl. Math., vol. 7, Interscience, New York, 1958. MR 22 \#8302.

9. R. Fllis, Locally compact transformation groups, Duke Math. J. 24 (1957), 119-125. MR 19, 561.

10. A. Grothendieck, Critères de compacité dans les espaces fonctionnels généraux, Amer. J. Math. 74 (1952), 168-186. MR 13, 857. 
11. C. J. Knight, W. Moran and J. S. Pym, The topologies of separate continuity. I, Proc. Cambridge Philos. Soc. 68 (1970), 663-671. MR 42 \#2422.

12. P. Milnes, Compactifications of semitopological semigroups, J. Austral. Math. Soc. 15 (1973), 448-503. MR 50 \#528.

13. T. Mitchell, Topological semigroups and fixed points, Illinois J. Math. 14 (1970), 630-641. MR 42 \#5245.

14. V. Pták, An extension theorem for separately continuous functions and its application to functional analysis, Czechoslovak Math. J. 14 (89) (1964), 562-581. MR 30 \#2334.

15. C. R. Rao, Invariant means on spaces of continuous or measurable functions, Trans. Amer. Math. Soc. 114 (1965), 187-196. MR 30 \#5128.

16. E. C. Titchmarsh, The theory of functions, 2nd ed., Oxford Univ. Press, London, 1939.

DEPARTMENT OF MATHEMATICAL SCIENCES, VIRGINIA COMMONWEALTH UNIVERSITY, RICHMOND, VIRGINIA 23284

DEPARTMENT OF MATHEMATICS, UNIVERSITY OF WESTERN ONTARIO, LONDON, ONTARIO, CANADA N6A 3K7 